\title{
On the 'magnetic' nature of electron transport barriers in tokamaks
}

\author{
S V Kasilov ${ }^{1}$, D Reiter ${ }^{2}$, A M Runov ${ }^{3}$, W Kernbichler ${ }^{4}$ and M F Heyn ${ }^{4}$ \\ ${ }^{1}$ Institute of Plasma Physics, National Science Center 'Kharkov Institute of Physics and \\ Technology', ul. Akademicheskaya 1, Kharkov 61108, Ukraine \\ 2 Institut für Plasmaphysik, Forschungszentrum Jülich GmbH, EURATOM Association, \\ Trilateral Euregio Cluster, Jülich D-52425, Germany \\ ${ }^{3}$ Institut für Plasmaphysik, Teilinstitut Greifswald, EURATOM Association, \\ Greifswald D-17491, Germany \\ ${ }^{4}$ Institut für Theoretische Physik, Technische Universität Graz, Petersgasse 16, Graz A-8010, \\ Austria
}

Received 25 October 2001, in final form 4 January 2002

Published 31 May 2002

Online at stacks.iop.org/PPCF/44/985

\begin{abstract}
The formation of internal transport barriers in the vicinity of rational magnetic surfaces in tokamaks with braided magnetic fields is studied for a simplified model of the perturbed magnetic field with a broad spatial spectrum and a monotonous shear profile. The island overlap criterion is used to derive a condition for barrier formation. This condition links the amplitude and the spectral width of the perturbation with the shear parameter. Numerical experiments with the MHD Monte-Carlo code E3D, where the problem of plasma heat conductivity is solved in $3 \mathrm{D}$, confirm this formation of transport barriers in the case of a monotonous shear profile. Assuming that experimentally observed electron internal transport barriers are the result of local reduction of electron heat transport due to the magnetic field braiding, the amplitude and spectral width of magnetic perturbations are estimated for the tokamak RTP.
\end{abstract}

\section{Introduction}

In various tokamak experiments, internal electron transport barriers (ITBs) have been observed in the vicinity of rational magnetic surfaces (e.g. see [1]). The phenomenological analysis [2] using a model with a locally stepwise reduced electron heat conductivity around the rational surfaces describes these observations quite well. One possible reason for the formation of ITBs is the local reduction of the anomalous transport caused by magnetic field perturbations. The formation of transport barriers in the ergodic divertor where the artificial 'anomalous' transport due to magnetic field braiding is dominant has been observed experimentally [3] and modelled numerically $[3,4]$. The role of magnetic perturbations in the formation of ITBs has been discussed, in particular, in $[5,6]$. The fact that a decrease of the shear parameter increases 
the distance between magnetic islands and disjoints them has been used as an explanation for the formation of experimentally observed transport barriers in the vicinity of the shear reversal point if the rotational transform approaches the low-order resonance value but does not cross it in this point. At the same time, it was stated in [5] that near-integer values of $q$ do not play a positive role in the case of a monotonous $q$ profile. In a recent analysis of the possibility of barrier formation in the case of a monotonous $q$ profile using a 'tokamap' model for the perturbed magnetic field [7], barriers have been identified near broken KAM surfaces with 'most noble' values of the rotational transform.

In this study, the formation of transport barriers is modelled using a simplified magnetic field with broad poloidal and toroidal spatial spectra of the perturbation field. This model is introduced in section 2. For such field spectra which are saturated exponentially with increasing angular wavenumbers, the formation of ITBs near low-order rational magnetic surfaces is shown in the case of a monotonous profile of $\iota=1 / q$. The origin of the barriers is connected with the fact that the distribution of the rational numbers over the real axis is not uniform. This fact has been used in $[8,9]$ to show the appearance of transport barriers in the presence of the electrostatic ion temperature gradient mode $\left(\eta_{\mathrm{i}}\right.$-mode) turbulence. In this paper, the increased distance between a low-order rational number and its nearest neighbouring rational numbers leads to the local reduction of the Chirikov overlap criterion below one. A simplified analysis using the overlap criterion is presented in section 2 where the criterion of barrier formation, equation (6), is derived following this simplified analysis. In section 3, this criterion is derived in a more rigorous way, with the help of a canonical perturbation theory. In section 4, the role of second harmonics of the perturbed Hamiltonian is estimated. Unlike the main harmonics of the perturbation field, these harmonics are resonant inside the 'barrier' region where they may overlap and cause a field line diffusion. However, the rate of this diffusion, as estimated in section 5, is reduced compared to the field line diffusion rate in the region outside the 'barrier'. In section 6, the analysis is verified through the numerical computation of the plasma temperature profile around the 'barrier' region using the 3D Monte-Carlo fluid code E3D [10]. The results of this computation are also compared in this section with the predictions of the Rechester-Rosenbluth model [11].

The criterion of barrier formation (6) can be used as an equation that links the amplitude and spectral width of the perturbation magnetic field with experimentally observed anomalous electron heat diffusivity. This is of special interest for cases in which neither the amplitude nor the spectrum is measured experimentally. The analytical formulae for the heat diffusion coefficient in a braided magnetic field, the Rechester-Rosenbluth formula in this case, provide a further equation. So, one can solve for the two unknown quantities; the perturbation amplitude $\epsilon$ and the poloidal spectral width $\bar{m}$ which defines the perpendicular scale of perturbations.

Thus, the information about the amplitude and the spectral width of magnetic perturbations can be obtained from experimental measurements of profiles of the electron temperature and of the safety factor in the case where the electron transport barrier is formed near the threshold of barrier formation. This seems to be the case in [1]. For this experiment, the spectral width and the perturbation amplitude are obtained with the help of the equation set given in section 7 . Finally, the results are discussed in section 8.

\section{Magnetic field model}

Here, the problem is solved using a simplified problem geometry represented by a straight periodic cylinder where the azimuth $\theta$ is the poloidal angle and the coordinate along the $z$-axis is the toroidal angle $\varphi \equiv z / R_{0}$, with $2 \pi R_{0}$ being the cylinder period. The main magnetic field is assumed to have constant shear, $\iota \equiv 1 / q=r$, where $0<r<1$ is a dimensionless radius. 
The perturbation field is derived from a vector potential of the form

$$
\boldsymbol{A}=\boldsymbol{e}_{z} A_{z}, \quad A_{z} \equiv A_{\varphi}=\frac{\epsilon r B_{\varphi}}{\bar{m}} \sum_{m=-M}^{M} \sum_{n=-N}^{N} a_{m, n} \sin \left(m \theta-n \varphi+\alpha_{m n}\right),
$$

with $B_{\varphi}=$ const and $\alpha_{m, n}$ are constant phase values, and $M$ and $N$ satisfy $M \geqslant \bar{m}$ and $N \geqslant \bar{n}$, respectively. Two kinds of spectra are considered in the following: a 'simplified' spectrum with $a_{m, n}=1$ and $M=N=\bar{m}=\bar{n}$, and a 'realistic' spectrum with

$$
a_{m, n}=\exp \left(-\frac{m^{2}}{\bar{m}^{2}}-\frac{n^{2}}{\bar{n}^{2}}\right)
$$

The 'simplified' spectrum is used below in analytical studies, and both kinds of spectra are used in the numerical modelling of heat conductivity. The perturbation field is obtained in the form

$$
\frac{B_{r}}{B_{\varphi}}=\sum_{m=-M}^{M} \sum_{n=-N}^{N} b_{m, n} \cos \left(m \theta-n \varphi+\alpha_{m, n}\right), \quad b_{m, n}=\epsilon \frac{m}{\bar{m}} a_{m, n} .
$$

In order to study the transition of such a system between regular and stochastic behavior using the Chirikov island overlapping criterion, one needs to estimate the size of the island structure near a given resonant magnetic surface with $\iota_{0}=n_{0} / m_{0}$. One should take into account all harmonics with certain helicity, $m=k m_{0}, n=k n_{0}$, where $k= \pm 1, \pm 2, \ldots$ The equation for the magnetic surfaces takes the following form:

$\frac{1}{R_{0}} \int_{0}^{r} \mathrm{~d} r^{\prime} r^{\prime}\left(\frac{n_{0}}{m_{0}}-\iota\left(r^{\prime}\right)\right)+\sum_{k} \frac{r}{k m_{0}} b_{k m_{0}, k n_{0}} \sin \left(k\left(m_{0} \theta-n_{0} \varphi\right)+\alpha_{k m_{0}, k n_{0}}\right)=$ const.

For an estimation of the sum in the second term in (4), it is assumed that the constant phase values $\alpha_{m, n}$ are distributed randomly. Using the estimation of the sum (43) in appendix B, one obtains for this second term $r b_{m_{0}, n_{0}} m_{0}^{-1}\left(2 \bar{m} / m_{0}\right)^{1 / 2} \log ^{1 / 2}\left(\bar{m} / m_{0}\right)$. Thus, for small perturbation amplitudes $\epsilon$, the island width is given as

$$
\delta r_{m_{0}, n_{0}} \approx 2\left(\frac{R_{0}\left|b_{m_{0}, n_{0}}\right|}{\left|m_{0} \iota^{\prime}\right|}\right)^{1 / 2}\left(\frac{2 \bar{m}}{m_{0}} \log \left(\frac{\bar{m}}{m_{0}}\right)\right)^{1 / 4}, \quad \iota^{\prime} \equiv \frac{\mathrm{d} \iota}{\mathrm{dr}}=1 .
$$

The radial position of a resonant surface is $r_{m_{0}, n_{0}}=n_{0} / \iota^{\prime} m_{0}=n_{0} / m_{0}$. Therefore, the particular chain of islands covers the radial interval $r_{m_{0}, n_{0}}-\delta r_{m_{0}, n_{0}}<r<r_{m_{0}, n_{0}}+\delta r_{m_{0}, n_{0}}$. A function $I(r)$ is defined so that it is equal to 1 if $r$ satisfies this condition for at least one mode $\left(m_{0}, n_{0}\right) . I(r)$ is equal to zero otherwise. Therefore, in the regions where $I(r)=1$, one can expect stochastic transport due to island overlapping and the formation of ergodic magnetic field regions, while in the regions with $I(r)=0$ no islands are present and transport should not be increased, i.e. a transport barrier is formed.

In figures 1 and 2 , the function $I(r)$ is shown for two different values of the perturbation amplitude $\epsilon$ together with resonant radii $r_{m, n}$ corresponding to low-order resonant surfaces, $m \leqslant 4$, represented as vertical lines. Here, $R_{0}=1$ in all numerical examples. One can observe that with decreasing perturbation amplitude, gaps with no islands (up to the cut-off of the spectrum) appear and become broader. These gaps first appear in the vicinity of loworder resonant surfaces on both sides of the corresponding island chain. One can expect that regular magnetic surfaces are formed first in these regions when the perturbation amplitude is decreased. This is confirmed in figures 3 and 4 where the results of the Poincare mappings are compared with predictions of the overlapping analysis. For this mapping, up to 1000 poloidal revolutions have been performed for a set of field lines starting at $\varphi=0$ at 20 equidistant radial positions in the interval $0.4<r<0.6$. The field lines have been traced until they leave the considered radial interval. 


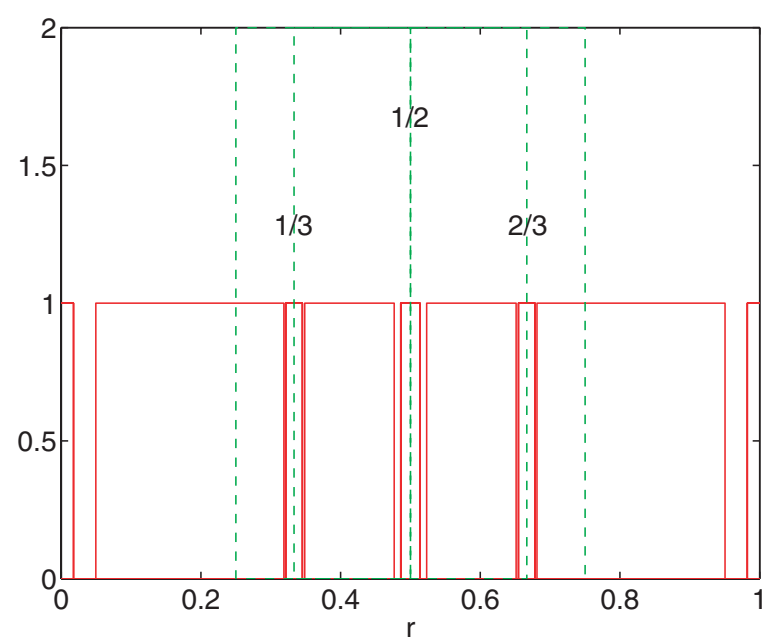

Figure 1. Function $I(r)$ for $\bar{m}=20$ and $\epsilon=10^{-4}$.

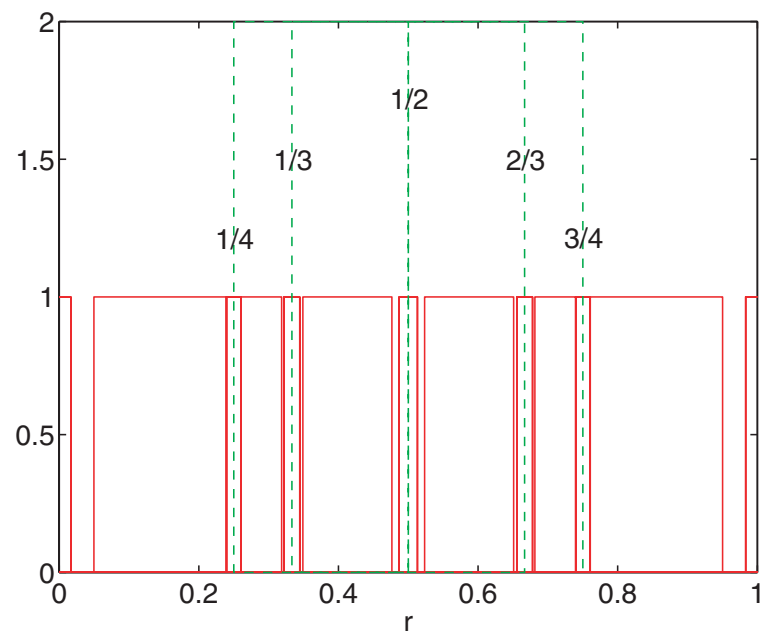

Figure 2. Same as figure 1 for $\epsilon=9 \times 10^{-5}$.

In figure 5 the inverse poloidal wavenumber, $1 / m$, is plotted as a function of the resonance position $r=n / m$ for resonant modes within the range $-M<m<M$ and $-N<n<N$ where $M=N=20$. One can observe that only the highest modes with small values of $1 / m$ are present in the vicinity of low-order resonances. With increasing $M$ and $N$ values, more resonances corresponding to higher-order modes should appear in a closer vicinity of the low-order resonance. However, these higher modes are not significant because they are exponentially small in the case of a 'realistic' spectrum.

In order to derive the threshold value of the perturbation amplitude for an ITB formation at some low-order resonance magnetic surface $\left(\iota\left(r_{0}\right)=n_{0} / m_{0}\right)$, one has to find the distances between the radial locations of the resonance $\left(m_{0}, n_{0}\right)$ and the nearest resonance with different helicity. The distance between $r_{0}=n_{0} / \iota^{\prime} m_{0}$ and the radius of the nearest resonant mode is $\Delta r=\Delta \iota /\left|\iota^{\prime}\right| \approx\left(\left|\iota^{\prime}\right| m_{0} M\right)^{-1} \sim\left(\left|\iota^{\prime}\right| m_{0} \bar{m}\right)^{-1}$. This estimate follows from inequalities (37) in 


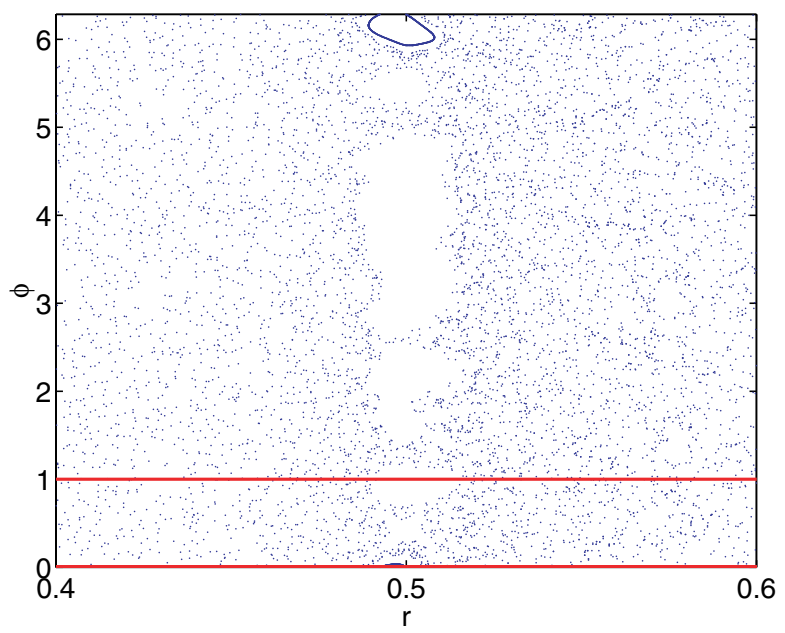

Figure 3. Poincaré plot of the magnetic field and function $I(r)$ for $\bar{m}=20$ and $\epsilon=2.6666 \times 10^{-4}$.

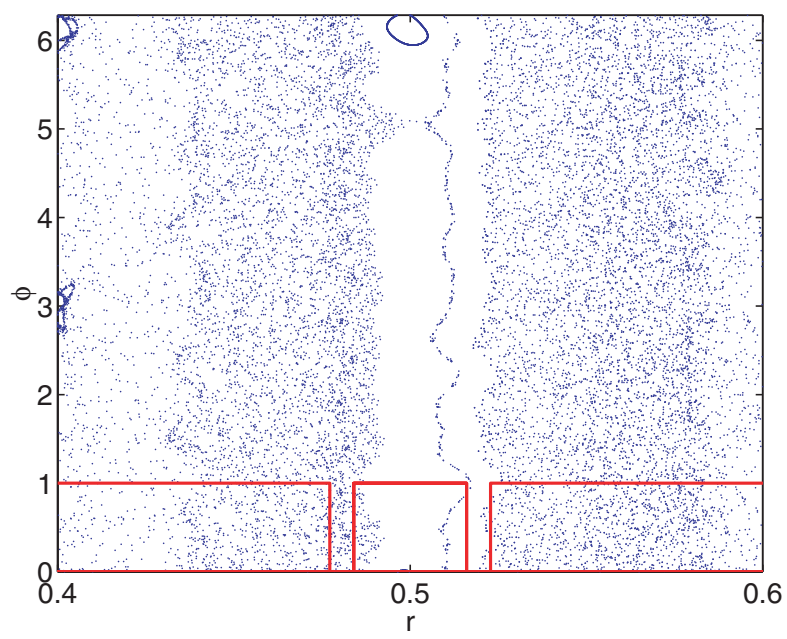

Figure 4. Same as figure 3 for $\epsilon=1.3333 \times 10^{-4}$.

appendix A and the assumption that $m_{0} \ll M \sim \bar{m}$. Comparing this distance to the radial size of low-order island structures (5), one obtains a criterion for the barrier formation:

$$
\begin{aligned}
\sigma_{\mathrm{b}} & =4 \times 2^{1 / 2}\left|\iota^{\prime}\right| R_{0} b_{m_{0}, n_{0}} \bar{m}^{5 / 2} m_{0}^{1 / 2} \log ^{1 / 2}\left(\frac{\bar{m}}{m_{0}}\right) \\
& \approx 4 \times 2^{1 / 2}\left|\iota^{\prime}\right| \in R_{0}\left(m_{0} \bar{m}\right)^{3 / 2} \times \log ^{1 / 2}\left(\frac{\bar{m}}{m_{0}}\right)<1,
\end{aligned}
$$

which links the perturbation amplitude $\epsilon$, the spectral width $\bar{m}$ and the shear $\iota^{\prime}$. This condition is much easily realized for low values of $m_{0}$. Therefore, ITBs should first form at the lowest order resonant magnetic surfaces. When comparing figures 1 and 2, one can notice that new 'barriers' corresponding to resonances $\frac{1}{4}$ and $\frac{3}{4}$ with $m_{0}=4$ appear during the transition from $\epsilon=10^{-4}$ to $\epsilon=9 \times 10^{-5}$. Estimating $\sigma_{\mathrm{b}}$ with $m_{0}=4, \bar{m}=20$ and $\epsilon=10^{-4}$, one obtains $\sigma_{\mathrm{b}}=0.51$. This is in agreement with (6) up to a numerical factor of order one. 


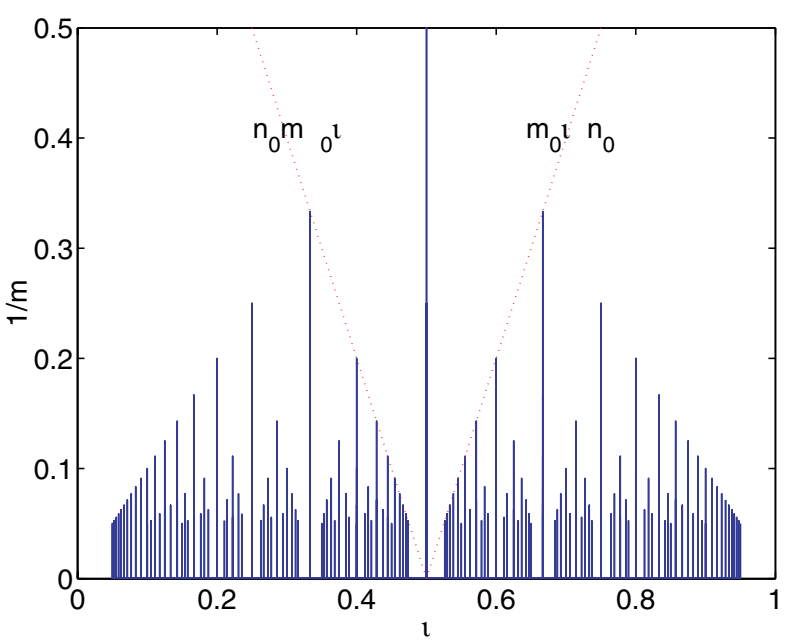

Figure 5. Locations of resonances.

\section{Resonance overlapping: Hamiltonian analysis}

By replacing the radius $r$ with the canonical momentum $I=r^{2} / 2$, the magnetic field line equations can be cast to the Hamiltonian form

$$
\frac{\mathrm{d} I}{\mathrm{~d} \varphi}=-\frac{\partial H(I, \theta, \varphi)}{\partial \theta}, \quad \frac{\mathrm{d} \theta}{\mathrm{d} \varphi}=\frac{\partial H(I, \theta, \varphi)}{\partial I},
$$

where the Hamiltonian is given as

$$
H=H_{0}(I)+H_{1}(I, \theta, \varphi), \quad H_{0}=\frac{\iota^{\prime}}{3}(2 I)^{3 / 2}, \quad H_{1}=-\frac{R_{0}}{B_{\varphi}} A_{\varphi},
$$

and $\varphi$ has the role of a time-like variable. For the 'simplified' spectrum one obtains

$$
H_{1}(I, \theta, \varphi)=\sum_{m, n=-\bar{m}}^{\bar{m}} H_{m, n} \sin \left(m \theta-n \varphi+\alpha_{m n}\right), \quad H_{m, n}=\varepsilon I^{1 / 2},
$$

where $\varepsilon=-\sqrt{2} \epsilon R_{0} / \bar{m}$. To eliminate the linear term in $\varepsilon$ in the Hamiltonian through the first iteration within the canonical perturbation theory (e.g. see [12]), one introduces the new momentum $\bar{I}$ and the new angle $\bar{\theta}$ with the help of the generating function $G(\bar{I}, \theta, \varphi)=$ $\bar{I} \theta+\varepsilon g(\bar{I}, \theta, \varphi):$

$$
I=\bar{I}+\varepsilon \frac{\partial g(\bar{I}, \theta, \varphi)}{\partial \theta}, \quad \bar{\theta}=\theta+\varepsilon \frac{\partial g(\bar{I}, \theta, \varphi)}{\partial \bar{I}} .
$$

The new Hamiltonian is

$$
\begin{aligned}
\bar{H} & =H(I, \theta, \varphi)+\varepsilon \frac{\partial g(\bar{I}, \theta, \varphi)}{\partial \varphi} \\
& =H_{0}(\bar{I})+\varepsilon\left(\Omega(\bar{I}) \frac{\partial g(\bar{I}, \theta, \varphi)}{\partial \theta}+\frac{\partial g(\bar{I}, \theta, \varphi)}{\partial \varphi}\right)+H_{1}(\bar{I}, \theta, \varphi)+H_{2},
\end{aligned}
$$


where $\Omega(\bar{I})=\partial H_{0}(\bar{I}) / \partial \bar{I}=\iota$ and $H_{2}=\mathrm{O}\left(\varepsilon^{2}\right)$. Thus, eliminating linear terms in $\varepsilon$ one obtains

$$
g(\bar{I}, \theta, \varphi)=\bar{I}^{1 / 2} \sum_{m, n=-\bar{m}}^{\bar{m}} \frac{\cos \left(m \theta-n \varphi+\alpha_{m n}\right)}{m \Omega(\bar{I})-n} .
$$

If the second of equations (10) can be resolved with respect to $\theta$ to give the unique solution $\theta=\theta(\bar{I}, \bar{\theta}, \varphi)$, then, up to linear order terms, the field lines will follow the invariant surface $\bar{I}=$ const which separates the space in radial direction. This is possible if $\partial \bar{\theta} / \partial \theta$ does not change its sign, yielding the condition

$\varepsilon\left|\frac{\partial^{2} g(\bar{I}, \theta, \varphi)}{\partial \bar{I} \partial \theta}\right| \approx \varepsilon\left|\bar{I}^{1 / 2} \frac{\mathrm{d} \Omega(\bar{I})}{\mathrm{d} \bar{I}} \sum_{m, n=-\bar{m}}^{\bar{m}} \frac{\sin \left(m \theta-n \varphi+\alpha_{m n}\right)}{(\Omega(\bar{I})-n / m)^{2}}\right|<1$.

Estimating in (13) the contribution of resonant terms at $\Omega=n_{0} / m_{0}$ (the number of these modes is $\left.2 \bar{m} / m_{0}\right)$ in the middle of the gap where $|\Omega(\bar{I})-n / m| \sim 1 / 2 m_{0} \bar{m}$, one obtains the condition

$$
\begin{aligned}
4 \times 2^{1 / 2} \varepsilon \bar{I}^{1 / 2} \frac{\mathrm{d} \Omega(\bar{I})}{\mathrm{d} \bar{I}}\left(m_{0} \bar{m}\right)^{2} \sqrt{\frac{\bar{m}}{m_{0}} \log \frac{\bar{m}}{m_{0}}} \\
=4 \times 2^{1 / 2}\left|\iota^{\prime}\right| \epsilon R_{0}\left(m_{0} \bar{m}\right)^{3 / 2} \log ^{1 / 2} \frac{\bar{m}}{m_{0}}=\sigma_{\mathrm{b}}<1,
\end{aligned}
$$

being identical to condition (6). Note that for the simplified spectrum in this section, the qualitative derivation of the criterion (6) given in the previous section leads to (14) without any additional factors. At the same time it is interesting to estimate the contribution from resonances for the harmonics with high wavenumbers located in the vicinity of the gap where $\Omega(\bar{I})<\left(n_{0} \bar{m}-1\right) / m_{0} \bar{m}$ or $\Omega(\bar{I})>\left(n_{0} \bar{m}+1\right) / m_{0} \bar{m}$. This quantity contains the sum (44) which is estimated in appendix B. Taking again the value of $\bar{I}$ in the middle of the 'barrier' region, $|\Omega(\bar{I})-n / m| \sim 1 / 2 m_{0} \bar{m}$, one finds $2 \varepsilon \bar{I}^{1 / 2}(\mathrm{~d} \Omega(\bar{I}) / \mathrm{d} \bar{I}) m_{0}^{3 / 2} \bar{m}^{5 / 2} \log ^{1 / 2} \bar{m}<1$, which is practically the same as (14).

The condition (6) is rather general and it is valid for spectra with perturbation amplitudes $b_{m, n}$ increasing in the long wavelength region $m \ll \bar{m}$ as $(m / \bar{m})^{K}$ where $K \geqslant 1$ and decreasing exponentially in the short wavelength region $m \gg \bar{m}$.

\section{Second harmonics}

Provided by (13), the difference between the old and new momenta is small, i.e. $|I-\bar{I}|$ is less than the width of the gap. Indeed, estimating the derivative of the sum (12) entering (10) in the same manner as sums (41) and (44) in appendix B, one gets $|I-\bar{I}| \sim \bar{I}^{1 / 2} \bar{m}^{3 / 2} m_{0}^{1 / 2} \sim$ $\sigma_{\mathrm{b}}\left(m_{0} \bar{m} \log ^{1 / 2}\left(\bar{m} / m_{0}\right) \mathrm{d} \Omega / \mathrm{d} I\right)^{-1} \ll \sigma_{\mathrm{b}}<1$. Quadratic and higher-order terms of this difference are retained in $H_{2}$. These terms contain now resonant harmonics on the combination frequencies $\Omega(\bar{I})=\left(n_{1}+n_{2}+\cdots\right) /\left(m_{1}+m_{2}+\cdots\right)$. These resonances are located inside the 'barrier' region (see figure 6), e.g. double resonances reduce the 'barrier' region by at least a factor of two. For estimates, one can retain only second harmonics in $\mathrm{H}_{2}$ containing two resonant denominators because they provide the highest contributions. This is the quadratic term in the expansion of $H_{0}(I)$. Putting $\theta=\bar{\theta}$ in the generating function $g$ used in (10), one gets

$$
H_{2}(\bar{I}, \bar{\theta}, \varphi) \approx \frac{\varepsilon^{2} \bar{I}}{2} \frac{\mathrm{d} \Omega(\bar{I})}{\mathrm{d} \bar{I}}\left(\sum_{m, n=-\bar{m}}^{\bar{m}} \frac{\sin \left(m \bar{\theta}-n \varphi+\alpha_{m n}\right)}{\Omega(\bar{I})-n / m}\right)^{2}
$$




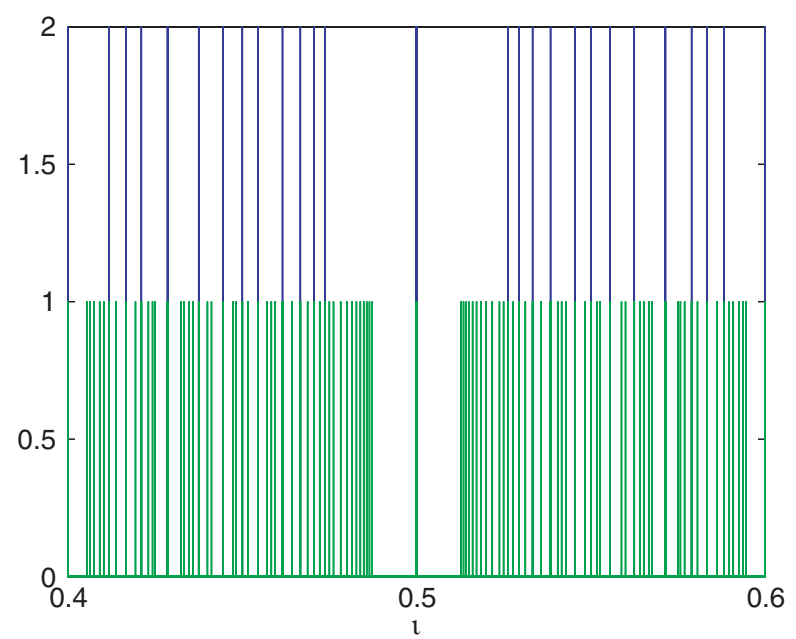

Figure 6. Locations of the main (vertical lines with amplitude equal to 2) and second harmonic (amplitude equal to 1) resonances.

First, one needs an estimate of that part of $\mathrm{H}_{2}$ which is independent of angles:

$$
\begin{aligned}
\left\langle H_{2}(\bar{I}, \bar{\theta}, \varphi)\right\rangle_{\bar{\theta}, \varphi} & \approx \frac{\varepsilon^{2} \bar{I}}{4} \frac{\mathrm{d} \Omega(\bar{I})}{\mathrm{d} \bar{I}} \sum_{m, n=-\bar{m}}^{\bar{m}} \frac{1}{(\Omega(\bar{I})-n / m)^{2}} \\
< & \frac{\varepsilon^{2} \bar{I}}{4} \frac{\mathrm{d} \Omega(\bar{I})}{\mathrm{d} \bar{I}}\left(\frac{2 \bar{m}}{m_{0}\left(\Omega(\bar{I})-n_{0} / m_{0}\right)^{2}}+\frac{\bar{m}^{2}}{\Omega(\bar{I})-\left(n_{0} \bar{m}-1\right) / m_{0} \bar{m}}\right. \\
& \left.-\frac{\bar{m}^{2}}{\Omega(\bar{I})-\left(n_{0} \bar{m}+1\right) / m_{0} \bar{m}}\right) .
\end{aligned}
$$

This part provides a frequency shift of the order of

$$
\Delta \Omega \equiv \frac{\mathrm{d}}{\mathrm{d} \bar{I}}\left\langle H_{2}(\bar{I}, \bar{\theta}, \varphi)\right\rangle_{\bar{\theta}, \varphi} \sim \frac{\varepsilon^{2} \bar{I}}{4}\left(\frac{\mathrm{d} \Omega(\bar{I})}{\mathrm{d} \bar{I}}\right)^{2} \bar{m}^{4} m_{0}^{2} \sim \sigma_{\mathrm{b}}^{2}\left(\bar{m} m_{0}\right)^{-1} \log ^{-1 / 2} \frac{\bar{m}}{m_{0}} \ll \sigma_{\mathrm{b}},
$$

which is small compared to $\Omega \sim 1$ inside the 'barrier' region. The radial scale length of this frequency shift is small too, $\mathrm{d} \Delta \Omega / \mathrm{d} \bar{I} \sim m_{0} \bar{m} \Delta \Omega \sim \sigma_{\mathrm{b}}^{2} \log ^{-1 / 2}\left(\bar{m} / m_{0}\right)$. The change of the magnetic field shear, $\mathrm{d} \Omega / \mathrm{d} \bar{I}$, will remain small as long as $\sigma_{\mathrm{b}} \ll 1$. Therefore, one can neglect this shift in further estimates.

The part of $H_{2}$ which alternates with angles is presented in the form

$\tilde{H}_{2}(\bar{I}, \bar{\theta}, \varphi) \equiv H_{2}(\bar{I}, \bar{\theta}, \varphi)-\left\langle H_{2}(\bar{I}, \bar{\theta}, \varphi)\right\rangle_{\bar{\theta}, \varphi}=\operatorname{Re} \sum_{m, n=-2 \bar{m}}^{2 \bar{m}} H_{m n}^{(2)} \exp (\mathrm{i}(m \bar{\theta}-n \varphi))$,

where $H_{m n}^{(2)}$ are complex amplitudes. As shown in appendix $\mathrm{C}$, the amplitudes of resonant harmonics located inside the barrier region, $\left(n_{0} \bar{m}-1\right) / m_{0} \bar{m}<n / m<\left(n_{0} \bar{m}+1\right) / m_{0} \bar{m}$, have the following order of magnitude:

$$
H_{m n}^{(2)} \sim \varepsilon^{2} \bar{I} \frac{\mathrm{d} \Omega(\bar{I})}{\mathrm{d} \bar{I}} m_{0}^{3 / 2} \bar{m}^{5 / 2} .
$$

The second-order resonances are distributed densely over $\bar{I}$ inside the 'barrier' region (see figure 6). The distance between them over $\bar{I}$ can be estimated as $\Delta \bar{I} \sim\left(4 \bar{m}^{2} \mathrm{~d} \Omega(\bar{I}) / \mathrm{d} \bar{I}\right)^{-1}$. 
Dividing the width of the secondary islands, $\delta \bar{I} \sim\left|H_{m n}^{(2)}\right|^{1 / 2}(\mathrm{~d} \Omega(\bar{I}) / \mathrm{d} \bar{I})^{-1 / 2}$, by this distance, one obtains the usual Chirikov overlapping criterion for second-order resonances:

$$
\sigma_{\mathrm{b}}^{(2)}=\frac{\delta \bar{I}}{\Delta \bar{I}} \sim \varepsilon \bar{I}^{1 / 2} \frac{\mathrm{d} \Omega(\bar{I})}{\mathrm{d} \bar{I}} m_{0}^{3 / 4} \bar{m}^{13 / 4} \sim \sigma_{\mathrm{b}}\left(\frac{\bar{m}}{m_{0}}\right)^{3 / 4} \log ^{-1 / 2} \frac{\bar{m}}{m_{0}}>1 .
$$

If $\sigma_{\mathrm{b}}$ is not too small, the overlapping condition (20) is realized due to a big factor $\left(\bar{m} / m_{0}\right)^{3 / 4}$. Note that $\sigma_{\mathrm{b}}$ cannot be very small, $\sigma_{\mathrm{b}}\left(\bar{m} / m_{0}\right)^{3 / 2}>1$, to ensure that ergodic magnetic field layers can exist at all. Comparing the second-order island widths with the width of the 'barrier' region, $\Delta \bar{I}_{\mathrm{b}}=\left(m_{0} \bar{m} \mathrm{~d} \Omega(\bar{I}) / \mathrm{d} \bar{I}\right)^{-1}$, one notices that these islands are small:

$$
\frac{\delta \bar{I}}{\Delta \bar{I}_{\mathrm{b}}} \sim \sigma_{\mathrm{b}}\left(\frac{m_{0}}{\bar{m}}\right)^{1 / 4} \log ^{-1 / 2} \frac{\bar{m}}{m_{0}} \ll \sigma_{\mathrm{b}}<1 .
$$

Therefore, one can describe the transport inside the 'barrier' region in terms of local magnetic field line diffusion, like within ergodic magnetic field regions outside the barrier (see next section).

Note that for small enough perturbation amplitudes, such that the size of the main helicity, $n_{0} / m_{0}$, island structure is small compared to the barrier width, there exists a region with further reduced diffusion which is free also from the second-order resonances (see figure 6). In outer parts of this region (with respect to the main island chain), also third-order resonances would be important. This structure is self-similar, and the amplitudes of each next order resonance harmonics are smaller than the previous one. Finally, close to the main island chain, the Chirikov overlap criterion will not be fulfilled and one can expect intact KAM surfaces there.

\section{Quasilinear diffusion}

In the framework of quasilinear theory, the magnetic field lines described by the Hamiltonian (8) are diffusing along $\varphi$ over the momentum $I$ with the diffusion coefficient

$$
D_{\mathrm{QL}}=\frac{\pi}{2} \sum_{m, n=-\bar{m}}^{\bar{m}} m^{2}\left|H_{m, n}\right|^{2} \delta(m \Omega-n) .
$$

The corresponding magnetic field line diffusion coefficient in real space coordinates [13], $D_{\mathrm{st}}=r^{-2}\left(R_{0}^{2}+(\iota r)^{2}\right)^{-1 / 2} D_{\mathrm{QL}}$, is

$$
D_{\mathrm{st}}=\frac{\pi R_{0}}{2 \sqrt{1+\left(\iota r / R_{0}\right)^{2}}} \sum_{m, n=-\bar{m}}^{\bar{m}}\left|b_{m n}\right|^{2} \delta(\iota m-n) .
$$

In the following, one also needs the quasilinear estimate for the Kolmogorov length [13] which is given by

$$
L_{\mathrm{K}}=\left(\frac{R_{0}}{\iota^{\prime} \tilde{m}}\right)^{2 / 3}\left(\frac{2}{D_{\mathrm{st}}}\right)^{1 / 3}, \quad \tilde{m}^{2}=\frac{\pi \sqrt{1+\left(\iota r / R_{0}\right)^{2}}}{2 D_{\mathrm{QL}}} \sum_{m, n=-\bar{m}}^{\bar{m}} m^{4}\left|H_{m, n}\right|^{2} \delta(m \Omega-n) .
$$

If the resonance overlapping is strong, such that modes with the same $m$ values and with neighbouring $n$ values overlap, the summation over $n$ is replaced by an integration giving

$$
D_{\mathrm{QL}}=\frac{\pi}{2} \sum_{m=-\bar{m}}^{\bar{m}} m^{2}\left|H_{m, m \Omega}\right|^{2} \approx \frac{\pi}{2} \int_{-\bar{m}}^{\bar{m}} \mathrm{~d} m m^{2}\left|H_{m, m \Omega}\right|^{2}=\frac{\pi}{3} \varepsilon^{2} I \bar{m}^{3} .
$$


For $D_{\text {st }}$ and $\tilde{m}^{2}$, one obtains

$$
D_{\mathrm{st}}=\frac{\pi \epsilon^{2} \bar{m} R_{0}}{3 \sqrt{1+\left(\iota r / R_{0}\right)^{2}}}, \quad \tilde{m}^{2}=\frac{3 \bar{m}^{2}}{5} \sqrt{1+\left(\iota r / R_{0}\right)^{2}},
$$

respectively. In addition to the simplified case, the integral can also be performed for the 'realistic' spectrum with $a_{m, n}$ given by (2) if one puts $M=N=\infty$. The result differs only by a numerical factor,

$$
D_{\mathrm{QL}}=\frac{\pi^{3 / 2} \varepsilon^{2} I \bar{m}^{3}}{8 \times 2^{1 / 2}\left(1+\Omega^{2}\right)^{3 / 2}},
$$

and for $D_{\text {st }}$ and $\tilde{m}^{2}$ one gets

$D_{\mathrm{st}}=\frac{\pi^{3 / 2} \epsilon^{2} \bar{m} R_{0}}{8 \times 2^{1 / 2}\left(1+\iota^{2}\right)^{3 / 2} \sqrt{1+\left(\iota r / R_{0}\right)^{2}}}, \quad \tilde{m}^{2}=\frac{4 \sqrt{1+\left(\iota r / R_{0}\right)^{2}}}{3\left(1+\iota^{2}\right)} \bar{m}^{2}$,

respectively.

In the case of weak overlapping when only a few neighbouring modes, e.g. $k$ modes, from the region outside the barrier overlap at a given radius, the delta function can be approximated by a Gaussian $(\bar{m} / \pi k)^{1 / 2} \exp \left(-\bar{m}(m \Omega-n)^{2} / k\right)$. Taking into account only $k$ terms contributing to the double sum in (22) and assuming $m \sim \bar{m}$, one obtains the estimate $D_{\mathrm{QL}} \sim \varepsilon^{2} I \bar{m}^{3}$. So, the degree of overlapping does not change the order of magnitude of $D_{\mathrm{QL}}$.

In a similar manner, one can estimate the quasilinear diffusion coefficient due to the second-order resonances in the 'barrier' region:

$$
D_{\mathrm{QL}}^{(2)} \sim \varepsilon^{4} \bar{I}^{2}\left(\frac{\mathrm{d} \Omega(\bar{I})}{\mathrm{d} \bar{I}}\right)^{2} m_{0}^{3} \bar{m}^{8} .
$$

Here the field lines are diffusing across the levels of the modified action $\bar{I}$. Taking the ratio of this coefficient to the diffusion coefficient outside the 'barrier' region, one obtains

$$
\frac{D_{\mathrm{QL}}^{(2)}}{D_{\mathrm{QL}}} \sim \sigma_{\mathrm{b}}^{2} \log ^{-1}\left(\frac{\bar{m}}{m_{0}}\right) .
$$

Thus, field line diffusion is significantly reduced inside the 'barrier' region near low-order rational resonances in the case of $\sigma_{\mathrm{b}} \ll 1$.

\section{MHD modelling of heat conductivity}

The full MHD transport properties of the chosen magnetic configurations have been studied numerically. The 3D Monte-Carlo code E3D [10] has been used to solve the problem of heat conductivity described by

$$
\frac{\partial T_{\mathrm{e}}}{\partial t}-\nabla \cdot\left(D_{\perp} \nabla T_{\mathrm{e}}+\left(D_{\|}-D_{\perp}\right) \boldsymbol{h} \boldsymbol{h} \cdot \nabla T_{\mathrm{e}}\right)=0,
$$

where $\boldsymbol{h}=\boldsymbol{B} / \boldsymbol{B}$ is the unit vector along the magnetic field. A unique feature of this code is the possibility to solve nonlinear advection-diffusion equations of highly anisotropic transport in an arbitrary magnetic field topology, including also partially or fully developed ergodicity. This is achieved by a combination of a bi-cubic spline cell mapping technique and of a MonteCarlo solution method. See [10] for details and an extensive analysis of the numerical precision achievable even for ergodic force fields. 
In the particular application for this paper, the stationary problem of heat propagation from a constant source at the inner boundary located at $r=0.4$ to an outer surface (the 'wall') located at $r=0.6$ is studied. The boundary condition at the wall is set to $T_{\mathrm{e}}=0$. A constant input heat flux $Q=40 \mathrm{~W}$ is prescribed at the inner boundary. The nonlinear dependence of the parallel heat conductivity coefficient on the plasma temperature is not taken into account in this case, and the background plasma temperature is chosen as $T_{\mathrm{e}}=25 \mathrm{eV}$. A fixed plasma density of $n_{\mathrm{e}}=10^{13} \mathrm{~cm}^{-3}$ is assumed. All length scales are measured in centimetres. The 'intrinsic' perpendicular heat diffusion coefficient $D_{\perp}=23 \mathrm{~cm}^{2} \mathrm{~s}^{-1}$ is taken $1.45 \times 10^{9}$ times smaller than the parallel diffusion coefficient $D_{\|}$. The magnetic field parameters are $\bar{m}=\bar{n}=10$ and $M=N=20$.

The results of E3D modelling are presented in figures 7-10 where the radial profiles of temperature averaged over both the poloidal and the toroidal angles are shown. Figures 7 and 8 correspond to the 'simplified' model of the magnetic field with $\bar{m}=20$ while figures 9 and 10

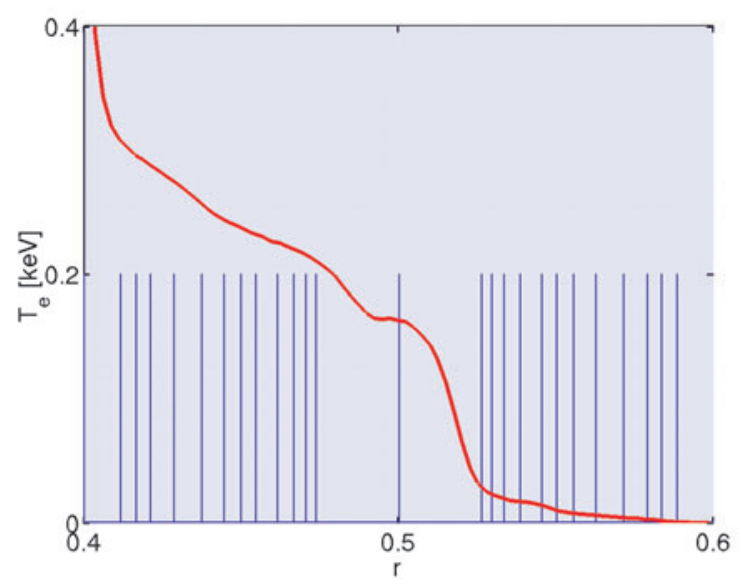

Figure 7. Temperature profile for 'simplified' model with $\epsilon=2.6666 \times 10^{-4}\left(\sigma_{\mathrm{b}}=0.58\right)$.

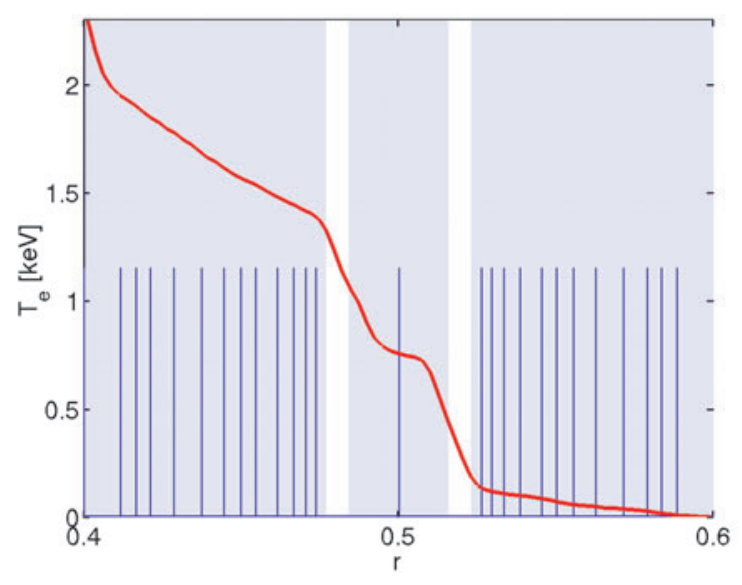

Figure 8. Same as figure 7 for $\epsilon=1.3333 \times 10^{-4}\left(\sigma_{\mathrm{b}}=0.29\right)$. 


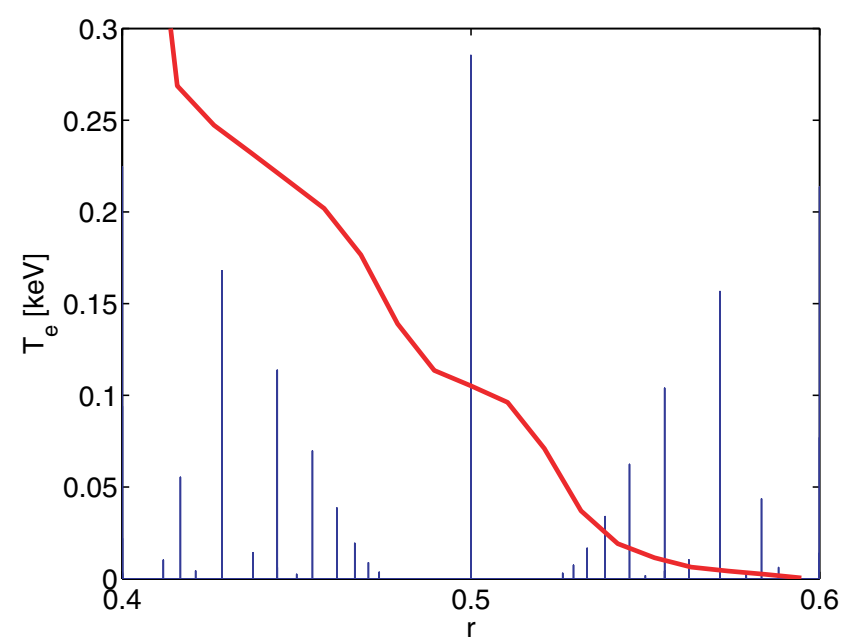

Figure 9. Temperature profile for 'realistic' model with $\epsilon=10^{-3}\left(\sigma_{\mathrm{b}}=0.64\right)$.

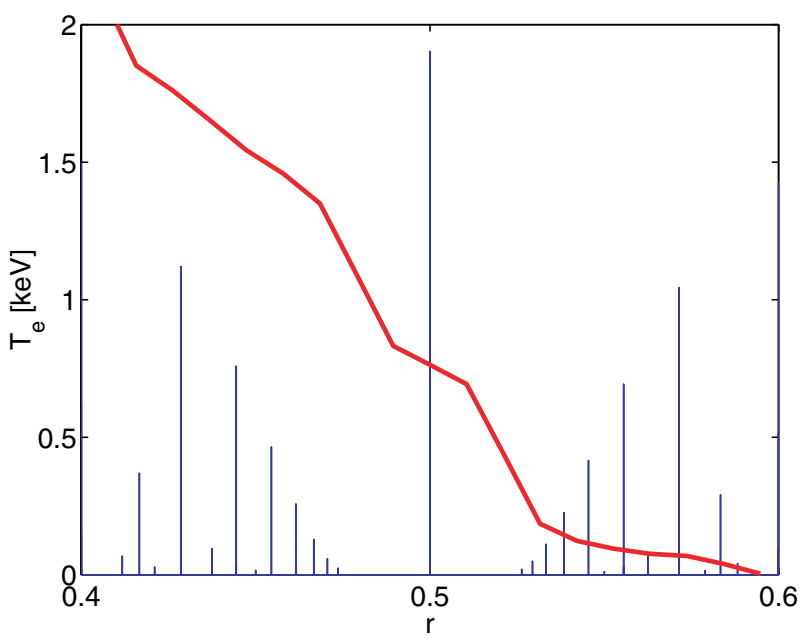

Figure 10. Same as figure 9 for $\epsilon=5 \times 10^{-4}\left(\sigma_{\mathrm{b}}=0.32\right)$.

correspond to the 'realistic' model with $\bar{m}=\bar{n}=10$ and $M=N=20$, respectively. The radial positions, $n / \iota^{\prime} m$ and amplitudes, $a_{m, n}$, of resonant modes are shown in these figures with vertical lines. The results are achieved by taking the magnetic field 'as it is', i.e. without any stochastic parameterization such as it would be introduced by 'magnetic field diffusivities'.

Shaded areas in figures 7 and 8 represent 'ergodic' regions where $I(r)=1$. In all the cases, transport due to magnetic field braiding is dominant since for the unperturbed magnetic field case, $\epsilon=0$, the temperature profile is close to a linear one. In that case, one gets a temperature value at the inner boundary of $7.3 \mathrm{keV}$. From the distribution of spectral modes, it is easy to identify the regions where barriers are formed. The calculated temperature gradient is significantly higher in these regions than in the 'ergodic' shaded regions. 'Barrier' regions are separated by the 'plateau' region with a very small temperature gradient. This region is formed 
at the place of the island chain corresponding to the 'main' helicity resonance $m_{0}=2$ and $n_{0}=1$. The overlapping condition is marginal in the cases considered here. With decreasing $\sigma_{\mathrm{b}}$, the temperature gradient in 'barrier' regions is increasing with respect to the gradient in ergodic magnetic field regions.

In figures 11 and 12 the effective 'diffusion' coefficient computed from the averaged temperature profile and normalized to $D_{\perp}$,

$$
\frac{D_{\text {eff }}}{D_{\perp}}=-\frac{Q}{4 \pi^{2} R_{0} r D_{\perp}}\left(\frac{\mathrm{d} T_{\mathrm{e}}}{\mathrm{d} r}\right)^{-1},
$$

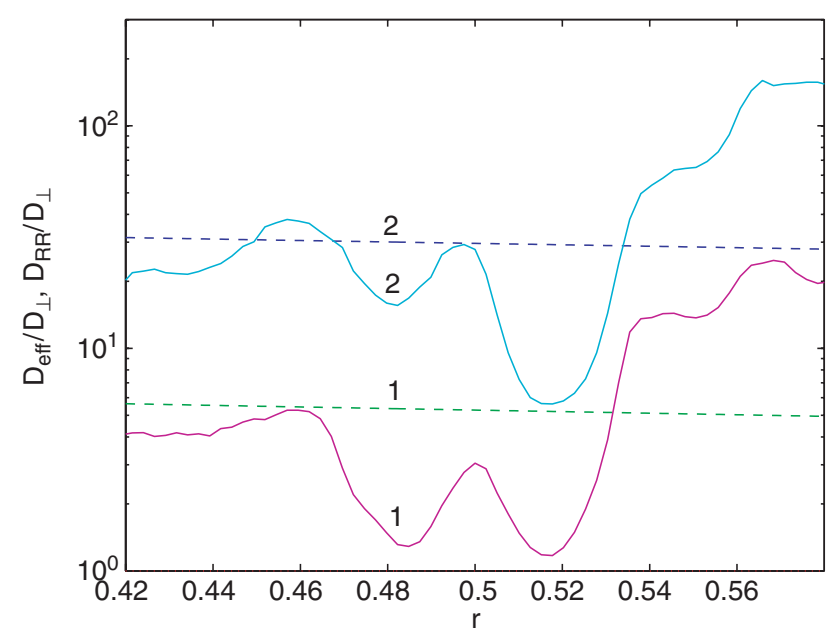

Figure 11. Normalized effective diffusion $D_{\text {eff }} / D_{\perp}(-)$ and the Rechester-Rosenbluth (- - - -) coefficients for the 'simplified' magnetic field spectrum. Curves $1-\epsilon=1.3333 \times 10^{-4}$, curves $2-\epsilon=2.6666 \times 10^{-4}$.

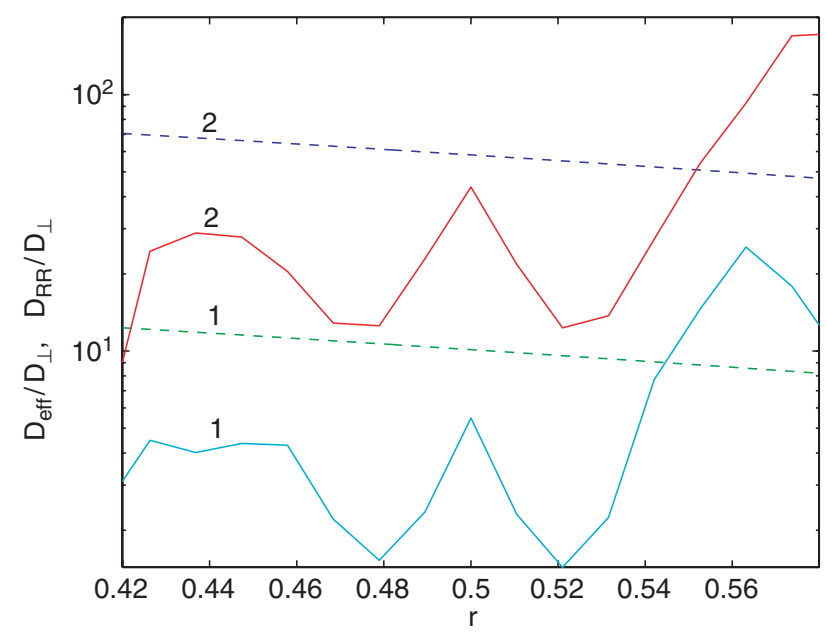

Figure 12. Normalized effective diffusion $D_{\text {eff }} / D_{\perp}(-)$ and the Rechester-Rosenbluth (- - - -) coefficients for the 'realistic' magnetic field spectrum. Curves $1-\epsilon=10^{-3}$, curves $2-\epsilon=5 \times 10^{-4}$. 
is compared to the normalized Rechester-Rosenbluth diffusion coefficient [11]:

$$
\frac{D_{\mathrm{RR}}}{D_{\perp}}=\frac{D_{\mathrm{st}}}{L_{\mathrm{c}}} \frac{D_{\|}}{D_{\perp}}, \quad L_{\mathrm{c}}=\frac{1}{2} L_{\mathrm{K}} \log \frac{r^{2} D_{\|}}{\bar{m}^{2} L_{\mathrm{K}}^{2} D_{\perp}} .
$$

Here, $L_{\mathrm{c}}$ is the parallel correlation length. For the 'simplified' magnetic field model, $D_{\mathrm{st}}$ and $L_{\mathrm{K}}$ are given by (26) and (24), respectively. For the 'realistic' model, equation (28) should be used instead of (26).

One can see that effective diffusion coefficients scale according to the RechesterRosenbluth theory. The effective diffusion coefficient drops in the 'barrier' region. For both models smaller values of $\epsilon$ and, respectively, smaller values of $\sigma_{\mathrm{b}}$ result in a stronger relative reduction of the transport coefficient due to the magnetic field ergodization, to the level below the 'intrinsic' perpendicular diffusion coefficient $D_{\perp}$. This is in agreement with the scaling in (30). The increase of the effective diffusion coefficient for $r>0.53$ is due to the 'laminar' parallel diffusion along the field lines with a relatively short connection length [10].

Thus, the MHD modelling confirms the presence of transport barriers in the case of a monotonous $q$ profile.

\section{Spatial scale and amplitude of magnetic perturbations}

The very fact of formation of a transport barrier with given helicity allows us to estimate the spatial scale of the magnetic field perturbation. It is given by the spectral width $\bar{m}$. Here one can assume that the observed anomalous electron heat diffusion coefficient $\chi_{\mathrm{A}}$ is fully due to magnetic field fluctuations. For the experimental conditions of RTP [1], transport is in the Rechester-Rosenbluth regime, since, as it is checked below, the mean free path is smaller than the parallel correlation length. Thus, one can put $\chi_{\mathrm{A}}=D_{\mathrm{RR}}$. Using (33) with $D_{\mathrm{st}}$ and $\tilde{m}$ given by (26) where small terms containing $\iota$ are neglected, one can express the perturbation amplitude $\epsilon$ through $\bar{m}$ explicitly. Substituting this in the barrier formation condition (14), one obtains the estimate for spectral width $\bar{m}$ and Kolmogorov length $L_{\mathrm{K}}$ :

$$
\begin{aligned}
& \bar{m}<\frac{\pi^{2 / 3}}{8 \times 3^{1 / 2} 5^{1 / 6} q^{\prime} R_{0} n_{0}^{2}}\left(\frac{\chi_{\mathrm{A}}}{D_{\|}} \log \frac{r^{2} D_{\|}}{\bar{m}^{2} L_{\mathrm{K}}^{2} D_{\perp}}\right)^{-1 / 2}\left(\log \frac{\bar{m}}{m_{0}}\right)^{-2 / 3}, \\
& L_{\mathrm{K}}=2^{1 / 2}\left(\frac{5}{3}\right)^{1 / 4} q\left(\frac{R_{0}}{q^{\prime} \bar{m}}\right)^{1 / 2}\left(\frac{\chi_{\mathrm{A}}}{D_{\|}} \log \frac{r^{2} D_{\|}}{\bar{m}^{2} L_{\mathrm{K}}^{2} D_{\perp}}\right)^{-1 / 4},
\end{aligned}
$$

respectively. Here, $q^{\prime}=\mathrm{d} q / \mathrm{d} r$ and $m_{0}=n_{0} q$. On RTP [1], the formation of a barrier at the $q=3$ resonant surface with $m_{0}=3$ and $n_{0}=1$ has been observed, in particular. To describe this, one takes the following set of parameters: $R_{0}=72 \mathrm{~cm}, r=8 \mathrm{~cm}, B=24 \mathrm{kG}$, $n_{\mathrm{e}}=2.4 \times 10^{13} \mathrm{~cm}^{-3}, T_{\mathrm{e}}=600 \mathrm{eV}, \chi_{\mathrm{A}}=5 \times 10^{3} \mathrm{~cm}^{2} \mathrm{~s}^{-1}, q^{\prime}=0.375 \mathrm{~cm}^{-1}$. For the 'intrinsic' diffusion coefficient $D_{\perp}$, the neoclassical expression in the banana regime, $D_{\perp} \sim v_{\perp} q^{2} \rho_{\mathrm{e}}^{2}\left(r / R_{0}\right)^{-3 / 2}$, is used. Here, $v_{\perp}$ and $\rho_{\mathrm{e}}$ are the collision frequency and the electron Larmor radius, respectively. Solving (34) with direct iterations (for this purpose equation (34) should be treated as an equality) one obtains $\bar{m} \approx 73, L_{\mathrm{K}} \approx 1500 \mathrm{~cm}$ and $L_{\mathrm{c}} \approx 4400 \mathrm{~cm}$. The last quantity helps to verify the validity of the Rechester-Rosenbluth theory, $l_{\mathrm{c}} / L_{\mathrm{c}} \ll 1$, where $l_{\mathrm{c}}=v_{T_{\mathrm{e}}} / v_{\perp}$ is the mean free path (see [13]). One obtains $l_{\mathrm{c}} / L_{\mathrm{c}} \approx 0.61<1$, i.e. this theory is marginally applicable. Thus, for the perpendicular wavenumber of magnetic perturbations, $k_{\perp}=\bar{m} / r$, one obtains the estimate $k_{\perp} \leqslant 9.1 \mathrm{~cm}^{-1}$.

Using (24) and (28), one obtains for the field line diffusion coefficient $D_{\mathrm{st}} \approx 6.3 \times 10^{-8} \mathrm{~cm}$ and for the perturbation amplitude $\epsilon \approx 3.4 \times 10^{-6}$, respectively. Assuming $\bar{n}=\bar{m}$, this gives 
for the perturbation magnetic field, $B_{r} \sim \epsilon(\bar{m} \bar{n})^{1 / 2} B_{\varphi}$, the estimate $B_{r} / B_{\varphi} \sim 2.5 \times 10^{-4}$. Note that this estimate is valid for the isotropic magnetic field spectrum, as given in (1). This spectrum is a very weak function of the radius $r$. At the same time, as shown in appendix B, only resonant modes with very low $k_{\|}$contribute to the process of formation of the ergodic layer and of the transport barrier. Thus, if one assumes a strongly anisotropic spectrum with very low $k_{\|}$but high $k_{\perp}$ (as estimated above), the estimate for the perturbation magnetic field is different, $B_{r} \sim \epsilon \bar{m}^{1 / 2} B_{\varphi}$, which means $B_{r} / B_{\varphi} \sim 2.9 \times 10^{-5}$.

In order to associate the value of the perpendicular wavenumber with a certain scale, one has to compute the ratio of the electron plasma frequency to the speed of light, $\omega_{\mathrm{pe}} / c \approx$ $9.2 \mathrm{~cm}^{-1}$. This scale follows from the theory of $[14,15]$. One can notice that this value is close to the estimate of $k_{\perp}$ obtained in the above analysis. At the same time, the obtained estimate for the magnitude of the fluctuating field is within the maximum range reported in experiments (see, e.g. [16]).

\section{Discussion}

In this analysis, it has been shown that heat transport caused by magnetic perturbations with a broad spectrum over the perpendicular (poloidal) wavenumbers may exhibit the 'barrier' feature in the case of a monotonous profile of the safety factor. Simple magnetic field models with such perturbation magnetic fields show barrier properties similar to those observed experimentally. There are two main features of these models. First, the poloidal spectrum of the perturbation field is rather broad. Therefore, the ergodization of magnetic surfaces distinct from the low-order rational magnetic surfaces is achieved due to the overlapping of resonances corresponding to high poloidal wavenumbers. Second, the amplitudes of radial components of different Fourier modes, $b_{m, n}$, are increasing with poloidal wavenumber within a certain range (see equation (3). As a consequence, the width of islands formed by a particular mode does not decrease within this range with increasing poloidal wavenumber. This results in robustness of magnetic surfaces around the low-order rational magnetic surfaces. Such a feature is quite different from general observations of Hamiltonian systems with narrow poloidal spectrum where the broadest chaotic zones are formed around hyperbolic points of the main (low $m$ ) islands. In the case of a broad perturbation field spectrum considered here, after the overlapping of resonances with high $m$, much broader chaotic regions appear in addition to the chaotic zones formed earlier at the place of destroyed separatrices of the low $m$ islands, which are separated from these regions by 'barrier' zones.

Using a simplified analysis with an island overlap criterion as well as a more detailed Hamiltonian formalism, the criterion of barrier formation, equation (6), is derived. This analysis is confirmed through the Poincaré mapping as well as through the direct 3D modelling of heat transport using the Monte-Carlo fluid code E3D. Although the $q$ profile used in the analysis is decreasing with radius which is not typical for tokamaks, the results should not change if more realistic $q$ profiles are considered. This is due to the fact that only a local behavior of $q$ near low-order rational magnetic surfaces is responsible for the effect. The $q$ profile can be well approximated there by a linear function, as in the model which is used here. The sign of the derivative of $q$ does not enter the barrier formation criterion (equation (6)); therefore, the increasing $q$ profile should show similar properties as the decreasing one.

In the phenomenological model of [2] barriers were introduced in close neighbourhood of rational $q$ values: below or above, but never extending across nor sitting on these rational surfaces. In this analysis, barriers are located on the sides of rational surface too, while at the position of the rational surface radial transport is strong due to the presence of the island structure there. On the other hand, two barriers appear to be linked with a certain rational $q$ 
value in the present model, that is different from [2] where only one barrier is linked to the rational $q$ value.

The particular physical mechanism responsible for the occurrence of magnetic perturbations is not the subject of this paper. On the other hand, for the analysis presented in section 7 it is essential that the spatial spectrum of the perturbation is independent of the structure of the distribution of resonant modes over the radius. As discussed in [8,9], such spectra are possible if the superposition principle is valid for the spectral modes, i.e. the turbulence is in the quasilinear regime. This might not be the case for the fully developed turbulence, e.g. skin-depth turbulence considered in $[14,15]$, where the saturation is achieved due to island overlapping, i.e. the resonance location pattern influences the amplitudes of the modes. In this case, the transport in the ergodic magnetic field regions located between the 'barriers' cannot be described within quasilinear theory because island remnants will occupy a significant volume in these regions. On the other hand, the saturation of modes from the main (short-scale, $m \gg 1$ ) part of the magnetic field spectrum does not destroy the 'barriers' around the low-order resonant surfaces as long as overlapping does not occur for the modes with low $m$. For example, such a situation is possible if those modes are stable. Another possibility exists at the initial stage of instability development if the growth rate of the modes with low $m$ does not exceed the growth rate of the short-scale modes. In particular, this is true for the resistive ballooning instability $[17,18]$.

Indeed, the evolution of the island width for a mode with given $m$ and $n, \delta r_{m, n}$, is described in the long-mean-free-path regime by the following equation (see [17]):

$$
\frac{\mathrm{d} \delta r_{m, n}}{\mathrm{~d} t}=\frac{0.27 \Delta^{\prime} c^{2}}{4 \pi \sigma_{\|}\left(1-2.1 \varepsilon_{t}^{1 / 2}\right)}-\frac{1.23 c^{2} q^{3} p^{\prime}}{\sigma_{\|} \varepsilon_{t}^{3 / 2} B^{2} q^{\prime}} \frac{1}{\delta r_{m, n}},
$$

where $\sigma_{\|}$is the parallel plasma conductivity, $\varepsilon_{t}=r / R_{0}, p^{\prime}=\mathrm{d}(n T) / \mathrm{d} r$ and $\Delta^{\prime}$ is the standard tearing mode theory parameter. In (36) only the first coefficient depends on $m$ because $\Delta^{\prime}$ depends on $m$. However, at the initial stage of island growth when the $\delta r_{m, n}$ are still small, the second term is dominant. As a result, the size of the islands at this stage is independent of $m$, in accordance with the model of this paper. In course of time, overlapping will first start for the modes with high $m$ forming ergodic magnetic field regions separated by 'barriers'. Finally, the 'barrier' zones are destroyed when the sizes of the low-order islands (low $m=m_{0}$ ) become of the order of the widths of the corresponding 'barrier' zones, $\Delta r=q^{2} /\left(\left|q^{\prime}\right| m_{0} k_{\perp} r\right.$ ) (see section 2). Note that the temporal analysis of the electron temperature profile carried out in [1] has shown that internal transport barriers appear (or are enhanced) during oblique pellet injection and start to vanish after a finite time $t$. In particular, for the barrier located at the $q=3$ surface, $t \sim 1 \mathrm{~ms}$. Assuming that the growth of islands (at least those with low $m$ ) restarts after the pellet injection, and estimating $\delta r_{3,1}$ from (36) (neglecting the first term on the right-hand side) together with the approximation for $p^{\prime} \sim n T_{\mathrm{e}} / r$ and using the same parameters as in section 7, one obtains $\delta r_{3,1} \sim 0.36 \mathrm{~cm}$. From $\Delta r=\delta r_{3,1}$ follows the estimate $k_{\perp} \sim 2.8 \mathrm{~cm}^{-1}$. Note that this estimate is still of the same order of magnitude as the estimate in section 7 .

It is interesting to discuss the scaling of the barrier conditions (6) and (14) with size using only a minimum number of assumptions on the scaling of the turbulence spectrum. First, one can assume that the perpendicular scale of micro-turbulence is linked to some microscopic parameter, such as the skin depth $c / \omega_{\text {pe }}$, rather than to the size of the device. Then, with increasing size of the device, the poloidal spectral width is also increasing, $\bar{m}=k_{\perp} r \sim r$. A second assumption is that the magnitude of the fluctuating field, $B_{r} / B_{\varphi}$, does not scale with size. For an isotropic turbulence spectrum, $\bar{n} \sim \bar{m}$, this would mean that $\epsilon \bar{m}$ stays constant. In fact, this would also mean that the quasilinear diffusion coefficient (26) does not scale with size either. In the case of a strongly anisotropic spectrum discussed at the end of the previous 
section, a constant magnitude of the fluctuating field would mean $\epsilon \bar{m}^{1 / 2}$ being constant. This means that the quasilinear diffusion coefficient is increasing with size. However, in both cases the barrier formation conditions (6) and (14) are becoming more restrictive with increased size of the device (for a fixed value of $m_{0}, \sigma_{\mathrm{b}} \sim r^{1 / 2}$ in the first case and $\sigma_{\mathrm{b}} \sim r$ in the second case). Since RTP is a rather small device compared to modern tokamaks, and the barriers were observed for small values of $m_{0}$, one can conclude that for large tokamaks electron ITBs can be achieved either at the inner radii or in regions where shear is considerably reduced. In particular, conditions (6) and (14) can be realized most easily near the shear reversal point in the case of an operation with reversed shear [5,6]. Probably, in experiments on T-10 [6] the micro-turbulence is not the only source of magnetic perturbations, since in cases when the safety factor is crossing the low-order rational value near the shear reversal point, strong tearing mode activity is observed. At the same time, if such crossing does not occur, but, instead, the value of the safety factor in the reversal point just approaches the low-order rational number, the electron ITB formation is observed. This is in full agreement with the theory given here.

\section{Acknowledgments}

This work has been carried out within the associations of EURATOM with IPP FZ-Jülich, with IPP Greifswald and with the Austrian Academy of Sciences. The content of the publication is the sole responsibility of its publishers and it does not necessarily represent the views of the Commission or its services. The authors are grateful to Prof. K N Stepanov for useful discussions.

\section{Appendix A. Distance between resonances}

First, the minimum distance between the given irreducible fraction $r_{0}=n_{0} / m_{0}<1$ and all other possible fractions $r=n / m \leqslant 1$ different from $r_{0}$ should be estimated. Here, $m, n, m_{0}$ and $n_{0}$ are integers from the interval between 1 and $M$. This distance is limited by

$$
\frac{1}{m_{0} M} \leqslant \min _{1 \leqslant n \leqslant m \leqslant M}\left|r-r_{0}\right|=\min _{1 \leqslant n \leqslant m \leqslant M}\left|\frac{n m_{0}-m n_{0}}{m m_{0}}\right| \leqslant \frac{1}{m_{0}\left(M-m_{0}+1\right)} .
$$

The first inequality is obvious since the minimum value for $\left|n m_{0}-m n_{0}\right|$ is 1 and the maximum value for $m$ in the denominator is $M$. To prove the second inequality, one has to prove that a pair of numbers $m$ and $n$ exists such that

$$
n m_{0}-m n_{0}=1,
$$

where $m$ belongs to the interval $M-m_{0}+1 \leqslant m \leqslant M$. Indeed, if there exists any solution $m^{(0)}, n^{(0)}$ to equation (38), then the solution within the required interval is obtained as $m=m^{(0)}-l m_{0}, n=n^{(0)}-l n_{0}$ where $l$ is an integer number given by $l=\left[\left(m^{(0)}-M\right) / m_{0}\right]$ for $m^{(0)} \leqslant M$ and $l=\left[\left(m^{(0)}-M\right) / m_{0}\right]+1$ for $m^{(0)}>M$. Here, [...] denotes the integer part. Such a solution is constructed using a recurrence relation. Introducing $i_{k}=\left[m_{k} / n_{k}\right]$ where $k=0,1,2, \ldots$, one defines for $k>0$ new coefficients $m_{k}$ and $n_{k}$ as well as new unknowns $m^{(k)}$ and $n^{(k)}$ in equation (38) through the recurrence relations:

$$
n_{k-1}=m_{k}, \quad m_{k-1}=i_{k-1} m_{k}+n_{k}, \quad n^{(k-1)}=m^{(k)}, \quad m^{(k-1)}=i_{k-1} m^{(k)}+n^{(k)} .
$$

As a result, this equation is transformed to

$$
n^{(k)} m_{k}-m^{(k)} n_{k}=(-1)^{k} \text {. }
$$


Finally, for a certain $k$ value, one ends up with $n_{k}=1$. Then, the solution to equation (40) is, for example, $n^{(k)}=0, m^{(k)}=(-1)^{k+1}$. With the help of the recurrence relation (39), one can reconstruct $m^{(0)}$ and $n^{(0)}$. It should be mentioned that residual numbers of the division $m_{k} / n_{k}$ are never zero until $n_{k}=1$ if $m_{0} / n_{0}$ is an irreducible fraction, otherwise equation (40) would mean that the pair $n_{k-1}=m_{k}, m_{k-1}=i_{k} m_{k}$ has a common factor $m_{k}$ and the same factor will enter, finally, both $m_{0}$ and $n_{0}$. This means that $r=n / m$ is an irreducible fraction as well. It is easy to check that the solution to (38) is unique in the given interval.

\section{Appendix B. Estimation of sums of randomly phased harmonics}

Here, the expectation value of the maximum value of the sum of the harmonics with random phases,

$$
\xi=\xi(\vartheta)=\sum_{m=-k}^{k} \cos \left(m \vartheta+\alpha_{m}\right),
$$

where $\alpha_{m}$ are random constant phases, is estimated. For large values of $k$, this number is Gaussian distributed with a probability density $p(\xi)=\exp \left(-\xi^{2} / 2\left\langle\xi^{2}\right\rangle\right) / \sqrt{2 \pi\left\langle\xi^{2}\right\rangle}$ for $\xi \ll k^{2 / 3}$, where $\left\langle\xi^{2}\right\rangle=k$. Since the correlation length for $\xi(\vartheta)$ is $\Delta \theta \approx 2 \pi / k$, one can have $2 \pi / \Delta \theta=k$ independent samples of $\xi$ within the period. The estimate for the maximum expected $|\xi|$ value after $k$ independent samplings, $\xi_{\max } \gg \sqrt{k}$, is obtained integrating the tails of the Gaussian distribution:

$$
\frac{1}{k}=2 \int_{\xi_{\max }}^{\infty} \mathrm{d} \xi p(\xi) \approx \frac{1}{\xi_{\max }} \sqrt{\frac{2 k}{\pi}} \exp \left(-\frac{\xi_{\max }^{2}}{2 k}\right) .
$$

Since $k \gg 1$, one obtains approximately $\xi_{\max } \approx \sqrt{2 k \log k}$. The same estimate is valid for the sum of the form

$$
\xi=\xi(\vartheta)=\sum_{j=-k}^{k} \cos \left(m_{0} j \vartheta+\alpha_{j}\right),
$$

after the change $m_{0} \vartheta \rightarrow \vartheta$.

Now the variance of the following double sum containing all harmonics except those with helicity $n_{0} / m_{0}$ has to be estimated:

$$
\xi(\theta, \varphi)=\sum_{m, n} \frac{\sin \left(m \theta-n \varphi+\alpha_{m n}\right)}{(\Omega(\bar{I})-n / m)^{2}} .
$$

Averaging over random phases, one obtains

$$
\begin{aligned}
\left\langle\xi^{2}\right\rangle= & \frac{1}{2} \sum_{m, n} \frac{1}{(\Omega(\bar{I})-n / m)^{4}}<\frac{1}{2} \sum_{k=1}^{\infty}\left(\frac{1}{\left(\Omega(\bar{I})-\left(\bar{m} n_{0}+1\right) / m_{0} \bar{m}-k / \bar{m}^{2}\right)^{4}}\right. \\
& \left.+\frac{1}{\left(\Omega(\bar{I})-\left(\bar{m} n_{0}-1\right) / m_{0} \bar{m}+k / \bar{m}^{2}\right)^{4}}\right) \\
\approx & \frac{1}{2} \int_{0}^{\infty} \mathrm{d} k\left(\frac{1}{\left(\Omega(\bar{I})-\left(\bar{m} n_{0}+1\right) / m_{0} \bar{m}-k / \bar{m}^{2}\right)^{4}}\right. \\
& \left.+\frac{1}{\left(\Omega(\bar{I})-\left(\bar{m} n_{0}+1\right) / m_{0} \bar{m}-k / \bar{m}^{2}\right)^{4}}\right) \\
= & \frac{\bar{m}^{2}}{6}\left(\frac{1}{\left(\Omega(\bar{I})-\left(\bar{m} n_{0}-1\right) / m_{0} \bar{m}\right)^{3}}-\frac{1}{\left(\Omega(\bar{I})-\left(\bar{m} n_{0}+1\right) / m_{0} \bar{m}\right)^{3}}\right) .
\end{aligned}
$$


Here, replacing the double sum with a single one, it was assumed that outside the gap, resonances are spaced densely with a distance $\sim 1 / \bar{m}^{2}$ between them (see figure 5 ). This assumption somewhat overestimates the sum since the maximum density of simple fractions $\bar{m}^{2}$ is assumed instead of the average one $\bar{m}^{2} / 2$. In order to estimate the number of independent samples of $\xi(\theta, \varphi)$, equation (44), one notes that this quantity is two dimensional. On the other hand, introducing the helical angle $\theta_{\mathrm{h}}=\theta-\varphi / \Omega(\bar{I})$ one notices that $\xi\left(\theta_{\mathrm{h}}, \varphi\right)$ is fully correlated over $\varphi$. Indeed, only harmonics with $n / m-\Omega(\bar{I}) \sim 1 / m_{0} \bar{m}$ effectively contribute to (44). For the parallel wavenumber $n_{\|}=n-\Omega(\bar{I}) m=R_{0} k_{\|}$, this gives the estimate $n_{\|}=m(n / m-\Omega) \sim 1 / m_{0}<1$. Thus, one needs to take into account only the short correlation length over $\theta_{\mathrm{h}}$ which is $2 \pi / \bar{m}$, and the number of samples is $\bar{m}$, respectively. Therefore, using the arguments given in the previous estimate, one obtains the maximum estimate for $\xi(\theta, \varphi)$ as $\xi_{\max } \approx \sqrt{\left\langle\xi^{2}\right\rangle \log \bar{m}}$, where $\left\langle\xi^{2}\right\rangle$ is given by (45).

\section{Appendix C. Amplitudes of second harmonics}

The complex amplitudes entering (18) have the following form:

$$
\begin{aligned}
H_{m n}^{(2)}= & -\frac{\epsilon^{2} \bar{I}}{8} \frac{\mathrm{d} \Omega(\bar{I})}{\mathrm{d} \bar{I}} \sum_{m_{1}, n_{1}, m_{2}, n_{2}=-\bar{m}}^{\bar{m}} \delta_{m, m_{1}+m_{2}} \delta_{n, n_{1}+n_{2}} \frac{1}{\left(\Omega(\bar{I})-n_{1} / m_{1}\right)\left(\Omega(\bar{I})-n_{2} / m_{2}\right)} \\
& \times\left(\exp \left(\mathrm{i} \alpha_{m_{1} n_{1}}+\mathrm{i} \alpha_{m_{2} n_{2}}\right)+\exp \left(-\mathrm{i} \alpha_{-m_{1}-n_{1}}-\mathrm{i} \alpha_{-m_{2}-n_{2}}\right)\right. \\
& \left.-2 \exp \left(\mathrm{i} \alpha_{m_{1} n_{1}}-\mathrm{i} \alpha_{-m_{2}-n_{2}}\right)\right) .
\end{aligned}
$$

Note that only modes with $m>\bar{m}$ can be resonant in the 'barrier' region. Here and below only positive values of $m$ are considered. For such modes, only the terms with both $m_{1}>0$ and $m_{2}>0$ contribute to the sum in (46). Therefore, the random phases entering different exponents in (46) are independent, except the exponents of the same kind with interchanging summation indices $m_{1}$ and $m_{2}$ (and $n_{2}$ and $n_{2}$ ). Calculating the variance of $\left|H_{m n}^{(2)}\right|$, one obtains

$$
\begin{aligned}
\left\langle\left|H_{m n}^{(2)}\right|^{2}\right\rangle= & \frac{3 \epsilon^{4} \bar{I}^{2}}{16}\left(\frac{\mathrm{d} \Omega(\bar{I})}{\mathrm{d} \bar{I}}\right)^{2} \sum_{m_{1}, n_{1}, m_{2}, n_{2}=1}^{\bar{m}} \delta_{m, m_{1}+m_{2}} \delta_{n, n_{1}+n_{2}} \\
& \times \frac{1}{\left(\Omega(\bar{I})-n_{1} / m_{1}\right)^{2}\left(\Omega(\bar{I})-n_{2} / m_{2}\right)^{2}} .
\end{aligned}
$$

To use the results of appendix A, the wavenumbers $m_{1,2}$ and $n_{1,2}$ are re-numerated in the following way:

$m_{1,2}=m^{\left(0, j_{1,2}\right)}-m_{0} k_{1,2}, \quad n_{1,2}=n^{\left(0, j_{1,2}\right)}-n_{0} k_{1,2}, \quad k_{1,2}=0,1,2, \ldots$,

where $m^{(0, j)}$ and $n^{(0, j)}$ are the pair of numbers which satisfy

$$
m_{0} n^{(0, j)}-n_{0} m^{(0, j)}=j, \quad j=0, \pm 1, \pm 2, \ldots
$$

and $\bar{m}-m_{0}+1 \leqslant m^{(0, j)} \leqslant \bar{m}$. Such solutions are constructed in analogy with solutions from appendix A. In particular, $m^{(0,0)}=m_{0}\left[\bar{m} / m_{0}\right]$. These solutions have the property $m^{(0, j)}+m^{(0, l-j)}=m^{(0, l)}+m^{(0,0)}+i$ where $i$ is either 0 or 1 . In the following $i=0$ is assumed. One can check that combinations of $m_{1}$ and $m_{2}$ in the form (48) which correspond to the resonances inside the barrier region should satisfy $j_{1}+j_{2}= \pm 1$ depending on the position of the resonance with respect to $n_{0} / m_{0}$. One can use the sign ' + ' without consequences for the result. Then, presenting $m=m^{(0,1)}+m^{(0,0)}-k m_{0}$ and $n=n^{(0,1)}+n^{(0,0)}-k n_{0}$, one obtains in (47) $m_{1}=m^{\left(0, j_{1}\right)}-m_{0} k_{1}, n_{1}=n^{\left(0, j_{1}\right)}-n_{0} k_{1}, m_{2}=m^{\left(0,1-j_{1}\right)}-m_{0}\left(k-k_{1}\right)$, 
$n_{2}=n^{\left(0,1-j_{1}\right)}-n_{0}\left(k-k_{1}\right)$, and the summation is over $j_{1}=0,1,2, \ldots$ and $k_{1}=0,1, \ldots, k$. Using (49) one can present

$$
\begin{aligned}
& \frac{n_{1}}{m_{1}}-\frac{n_{0}}{m_{0}}=\frac{j_{1}}{m_{0}\left(m^{\left(0, j_{1}\right)}-m_{0} k_{1}\right)} \approx \frac{j_{1}}{m_{0}\left(\bar{m}-m_{0} k_{1}\right)} \approx \frac{j_{1}}{m_{0} \bar{m}}+\frac{j_{1} k_{1}}{\bar{m}^{2}}, \\
& \frac{n_{2}}{m_{2}}-\frac{n_{0}}{m_{0}} \approx \frac{1-j_{1}}{m_{0} \bar{m}}+\frac{\left(1-j_{1}\right)\left(k-k_{1}\right)}{\bar{m}^{2}},
\end{aligned}
$$

and, as a result, an estimate for the sum entering (47) is

$$
\begin{aligned}
\sum_{m_{1}, n_{1}, m_{2}, n_{2}=1}^{\bar{m}} \delta_{m, m_{1}+m_{2}} \delta_{n, n_{1}+n_{2}} \frac{1}{\left(\Omega(\bar{I})-n_{1} / m_{1}\right)^{2}\left(\Omega(\bar{I})-n_{2} / m_{2}\right)^{2}} & \\
\sim & \sum_{j_{1}=0}^{\infty} \sum_{k_{1}=0}^{k}\left(\Omega-\frac{n_{0}}{m_{0}}-\frac{j_{1}}{m_{0} \bar{m}}-\frac{j_{1} k_{1}}{\bar{m}^{2}}\right)^{-2} \\
& \times\left(\Omega-\frac{n_{0}}{m_{0}}-\frac{1-j_{1}}{m_{0} \bar{m}}-\frac{\left(1-j_{1}\right)\left(k-k_{1}\right)}{\bar{m}^{2}}\right)^{-2} \sim \frac{\bar{m}^{2}}{\left(\Omega-n_{0} / m_{0}\right)^{3}} \sim m_{0}^{3} \bar{m}^{5} .
\end{aligned}
$$

Estimating now $\left|H_{m n}^{(2)}\right|$ as a square root of the variance (47), one arrives at (19).

\section{References}

[1] Gorini J et al 2000 Plasma Phys. Control. Fusion 42 (suppl 5A) A161-8

[2] Hogeweij G M D et al 1998 Nucl. Fusion 38 1881-91

[3] Feron S and Ghendrich Ph 1997 24th EPS Conf. on Contr. Fusion and Plasma Phys. (Berchtesgaden, 9-13 June 1997) vol 21A, pp 185-7

[4] Becoulet M et al Preprint EUR-CEA-FC-1667

[5] Alladio F et al 1999 Plasma Phys. Control. Fusion 41 A323-32

[6] Razumova K A et al 2000 Plasma Phys. Control. Fusion 42 973-86

[7] Misguich J H 2001 Phys. Plasmas 8 2132-8

[8] Beklemishev A D and Horton W 1992 Phys. Fluids B 4 200-6

[9] Beklemishev A D and Horton W 1992 Phys. Fluids B 4 2176-80

[10] Runov A M et al 2001 Phys. Plasmas 8 916-30

[11] Rechester A B and Rosenbluth M N 1978 Phys. Rev. Lett. 40 38-41

[12] Lichtenberg A J and Lieberman M A 1992 Regular and Chaotic Dynamics 2nd edn (New York-BerlinHeidelberg-London-Paris-Tokyo-Hong Kong-Barcelona-Budapest: Springer)

[13] Krommes J A, Oberman C and Kleva R G 1983 J. Plasma Phys. 30 11-56

[14] Kadomtsev B B 1991 Nucl. Fusion 31 1301-14

[15] Smolyakov A I and Hirose A 1993 Phys. Fluids B 5 663-5

[16] Zweben S J and Taylor R J 1981 Nucl. Fusion 21 193-200

[17] Carrera R, Hazeltine R D and Kotschenreuter M 1986 Phys. Fluids 29 899-902

[18] Kuvshinov B N et al 1989 Fizika Plazmy (Sov. J. Plasma Phys.) 15 1160-3 (in Russian) 